\title{
Control of Teleoperation Systems Operating under Communication Line Failures
}

\author{
Mehmet Ismet Can Dede \\ Izmir Institute of Technology \\ Department of Mechanical Engineering \\ Urla/Izmir, Turkey 35430 \\ cdede002@fiu.edu
}

\author{
Sabri Tosunoglu \\ Florida International University \\ Department of Mechanical Engineering \\ Miami, Florida 33174 \\ tosun@fiu.edu
}

\begin{abstract}
While the robots gradually become a part of our daily lives, they already play vital roles in many critical operations. Some of these critical tasks include surgeries, battlefield operations, and tasks that take place in hazardous environments or distant locations such as space missions. In most of these tasks, remotely controlled robots are used instead of autonomous robots. This special area of robotics is called teleoperation. Teleoperation systems must be reliable when used in critical tasks; hence, all of the subsystems must be dependable even under a subsystem or communication line failure. Teleoperation controllers are designed to compensate for instabilities due to communication time delays. Modifications to the existing controllers are proposed to configure a controller that is reliable under communication line failures. Experimental studies are then conducted on limited- and unlimited-workspace teleoperation systems to verify the efficiency of the controllers proposed for each system.
\end{abstract}

\section{INTRODUCTION}

Teleoperation represents an application area where humans cannot achieve the job either because the task is too dangerous or needs to be carried out at a distant site from the main control location. Robots, which work in radioactive and hazardous environments, are examples to the robotics tasks that are very dangerous for the humans to accomplish.

Teleoperation systems are composed of a master and a slave subsystem. These subsystems cooperate to complete a task at sites that are either at distant places or at places that are hazardous for humans. The human operator uses a master system to send out demands. The slave system is driven by these demands. Depending on the information flow, teleoperation systems are usually called unilateral or bilateral. In unilateral teleoperation, no feedback is provided from slave to the master and slave is driven by the commands sent by the master. In bilateral teleoperation, any kind of feedback from the slave to the master can be sent. These feedback signals can be visual, or contain force, sound, position, temperature, or radiation information.

In force-feedback teleoperation, as it was the case in unilateral teleoperation, the slave system is driven by the commands sent from the master system. However, in this case, the slave system sends back the force-feedback information that it produces while interacting with the environment to the master system so that the operator has a feeling of the remote system's environment. Bilateral teleoperation systems are also investigated in two sublevels as limited- and unlimited-workspace teleoperation.

Teleoperation systems using serial or parallel slave manipulators with limited workspace are defined as limitedworkspace teleoperation. Telemanipulation of an industrial robot arm is a typical example to this type of teleoperation. Researchers working on the stabilization issue of the timedelayed teleoperation have also chosen to work with limitedworkspace teleoperators [1, 2, 3]. In limited-workspace teleoperation, generally master position and orientation information is mapped into the Cartesian position and orientation of the end-effector. Therefore, position tracking becomes a priority for the limited-workspace.

Teleoperation systems composed of a mobile platform or any unlimited-workspace slave is referred to as unlimitedworkspace teleoperation. Telemanipulation of any mobile robotic system whether it operates on ground, water or in air is categorized as unlimited-workspace teleoperation. In unlimited-workspace teleoperation, the position information from the master is generally mapped as velocity demand for the end-effector of the slave. The tracking priority is given to the velocity in unlimited-workspace teleoperations. JPL's Urbie Rover is an example to the unlimited-workspace teleoperation [4].

Most of the time, teleoperation systems are employed in critical missions. For example, TALON® EOD robot has been used in numerous critical military tasks in years [5]. These critical missions require the teleoperation system to be stable and dependable even under extraordinary conditions for continuous operation. Communication is the most common conditions to be listed as extraordinary.

In the past two decades, researchers have worked on compensation techniques to stabilize teleoperation systems as they experience time delays. Among the control techniques developed for this, Anderson and Spong [6] were the first ones to introduce scattering transforms to overcome instability due to time delays. Then Niemeyer and Slotine [1] further developed this concept into the wave variable technique.

The customary wave variable technique and the proposed additional components to this technique are presented in this paper. The position feedforward component is designed to compensate for position offsets that may form when 
communication losses occur. Stability is proposed to be maintained by the adaptive gain component for teleoperation systems with variable time delays.

These controllers are then examined on limited- and unlimited-workspace teleoperation systems under variable time delays. Finally, conclusions are provided with the future work towards configuring reliable teleoperation systems.

\section{TELEOPERATION CONTROLLERS}

In this section, initially, customary wave variable technique is presented. Later, the two additional components are described and the teleoperation block diagram is altered.

\section{A. Customary Wave Variable Technique}

The common shortcoming of the force-feedback teleoperation is the instability that the system undergoes when it experiences time delays in communications between the master and the slave. The magnitude of this time delay could be in the order of seconds, minutes, hours or days depending on the nature of teleoperation. This problem has been studied by many researchers, but Anderson and Spong were perhaps the first to use the wave variable method to control bilateral controllers [6]. Also, Niemeyer and Slotine [1], and Munir and Book [2] have implemented this method to teleoperation systems. Current studies are on the variable time-delayed teleoperation [2, 3]. Although the wave variable technique guarantees stability for the constant time delayed teleoperation, the system experiences instability when the time delay varies.

The block diagram in Fig. 1 below presents the wave variable technique in terms of the scattering transformation a mapping between the velocity and force signals, and the wave variables [1].

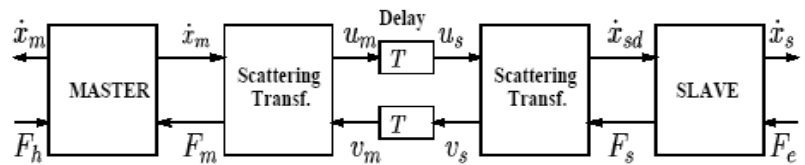

Fig. 1. Scattering transformation for teleoperation with constant time delay [1]

This transformation using the notation in [2] is described as follows:

$$
\begin{aligned}
& u_{s}=\frac{1}{\sqrt{2 b}}\left(b \dot{x}_{s d}+F_{s}\right) ; \quad u_{m}=\frac{1}{\sqrt{2 b}}\left(b \dot{x}_{m}+F_{m}\right) \\
& v_{s}=\frac{1}{\sqrt{2 b}}\left(b \dot{x}_{s d}-F_{s}\right) ; \quad v_{m}=\frac{1}{\sqrt{2 b}}\left(b \dot{x}_{m}-F_{m}\right)
\end{aligned}
$$

where $\dot{x}_{m}$ and $\dot{x}_{s}$ are the respective velocities of the master and the slave, $F_{h}$ is the torque applied by the operator, $F_{e}$ is the torque applied externally on the remote system, $F_{m}$ is the force reflected back to the master from the slave robot, $F_{s}$ is the force information sent from the slave to master, $\dot{x}_{s d}$ is the velocity derived from the scattering transformation at the slave side, $u$ and $v$ define the wave variables.

The power, $P_{i n}$, entering a system can be defined as the scalar product between the input vector $x$ and the output vector $y$. Such a system is defined to be passive if and only if the following holds:

$\int_{0}^{t} P_{\text {in }}(t)=\int_{0}^{t} x^{T} y d \tau \geq E_{\text {store }}(t)-E_{\text {store }}(0)$

where $E(t)$ is the energy stored at time $t$ and $E(0)$ is the initially stored energy. The power into the communication block at any time is given by

$P_{i n}(t)=\dot{x}_{m d}(t) F_{m}(t)-\dot{x}_{s d}(t) F_{s}(t)$

In the case of the constant communications delay where the time delay $T$ is constant,

$u_{s}(t)=u_{m}(t-T) ; \quad v_{m}(t)=v_{s}(t-T)$

Substituting these equations into (3), and assuming that the initial energy is zero, the total energy $E$ stored in communications during the signal transmission between master and slave is found as

$$
\begin{gathered}
E=\int_{0}^{t} P_{i n}(\tau) d \tau=\int_{0}^{t}\left(\dot{x}_{m d}(\tau) F_{m}(\tau)-\dot{x}_{s d}(\tau) F_{s}(\tau)\right) d \tau \\
=\frac{1}{2} \int_{0}^{t}\left(u_{m}^{T}(\tau) u_{m}(\tau)-v_{m}^{T}(\tau) v_{m}(\tau)+v_{s}^{T}(\tau) v_{s}(\tau)-u_{s}^{T}(\tau) u_{s}(\tau)\right) d \tau(5) \\
=\frac{1}{2} \int_{t-T}^{t}\left(u_{m}^{T}(\tau) u_{m}(\tau)+v_{s}^{T}(\tau) v_{s}(\tau)\right) d \tau \geq 0
\end{gathered}
$$

Therefore, the system is passive independent of the magnitude of the delay $T$. In other words, the time delay does not produce energy if the wave variable technique is used. Therefore, it guarantees stability for the constant timedelayed teleoperation.

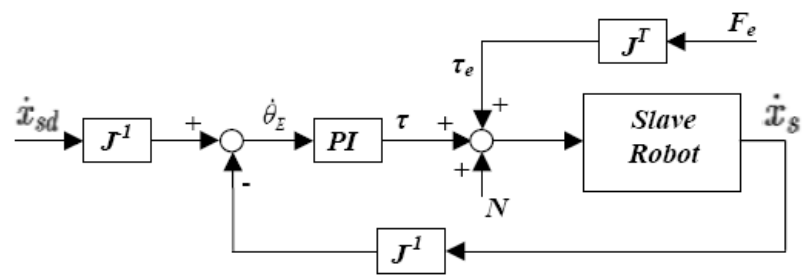

Fig. 2. Customary velocity control of the slave manipulator

The customary formation of the wave variable technique is illustrated in Fig. 1. The controller on the slave side is usually 
a velocity controller. A representation of the basic velocity controller block is shown in Fig. 2. The velocity error is calculated in Cartesian space and then translated into joint velocity errors using the inverse of the Jacobian matrix, $J$. A general type of proportional-integral control is applied to calculate the driving torque for each joint. The $N$ in the block diagram of Fig. 2 represents the feedforward torque input to counteract the centrifugal, Coriolis and gravitational forces. Calculation of the errors in Cartesian space enables the usage of unlike masters and slaves.

The system dynamics is written for teleoperation with the customary wave variable control as:

$$
M_{m} \ddot{x}_{m}+B_{m} \dot{x}_{m}=F_{h}-F_{m} ; \quad M_{s} \ddot{x}_{s}+B_{s 1} \dot{x}_{s}=F_{s}-F_{e}
$$

where $M_{m}$ and $M_{s}$ are the respective inertias of the master and the slave, and $B_{m}$ and $B_{s 1}$ are the master and slave damping respectively. The slave force can be formulated as;

$$
F_{s}(t)=K_{I} \int_{0}^{t}\left(J^{-1} \dot{x}_{s d}-J^{-1} \dot{x}_{s}\right) d s+K_{v}\left(J^{-1} \dot{x}_{s d}-J^{-1} \dot{x}_{s}\right)
$$

where $K_{I}$ and $K_{v}$ are the integral and proportional gain respectively.

This controller produces acceptable system response when the wave variable technique is active but the communication is never lost between the master and the slave. When the communication is lost for limited periods, an offset between the master and slave position tracking occurs.

\section{B. Position Drift Compensator for Wave Variable Technique}

A feedforward position demand is proposed to modify the wave variable technique to compensate for the offsets mentioned above. This demand is sent from the master system directly to the slave without integrating it in the scattering transform. This modification does not include a force feedforward component as in [7] because no drifts have been observed between the slave and the master force information in experimental studies [8]. The block diagram of the proposed algorithm is given in Fig. 3.

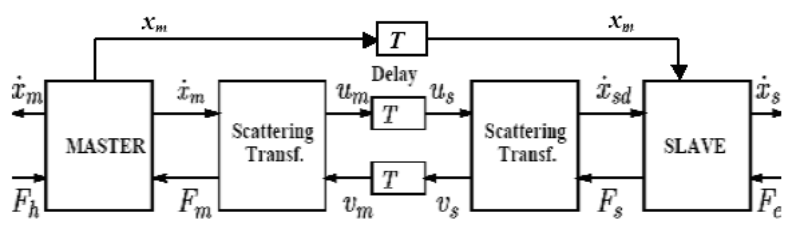

Fig. 3. Offset compensation for the wave variable technique

The slave controller block diagram is also modified to comply with the new setting of the wave variable technique. As observed in Fig. 4, the position error is calculated in the joint space. The motion demand from the master received in Cartesian space is transformed into the joint space by using the inverse of the Jacobian, $J$, and the inverse kinematics, $I K$. Later, the demand in joint space is compared to the joint sensor readings to form joint motion errors. This type of controller is of course feasible for those manipulators for which the inverse kinematics solutions are easy to obtain. Fortunately, almost all of the industrial manipulators are of this kind [9].

After the modification to the wave variable technique, the system dynamics is written as

$M_{s} \ddot{x}_{s}+B_{s 1} \dot{x}_{s}=F_{s}-F_{e} ; \quad M_{m} \ddot{x}_{m}+B_{m} \dot{x}_{m}=F_{h}-F_{m}$

where

$F_{s}=K_{d}\left(J^{-1} \dot{x}_{s d}-J^{-1} \dot{x}_{s}\right)+K_{p}\left(I K\left(x_{m}(t-T)\right)-I K\left(x_{s}\right)\right)(9)$

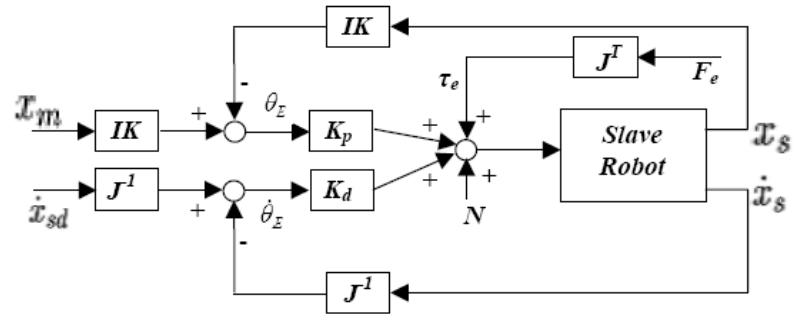

Fig. 4. Modified controller for the slave system for the wave variable technique with position feedforward component

The control gains used in customary wave variable slave controller may be applied to the modified version. Therefore, the modified controller's gain magnitudes of $K_{d}$ and $K_{p}$ may be selected as equal to the magnitude of $K_{v}$ and $K_{I}$.

\section{Wave Variable Technique with Adaptive Gain}

The block diagram in Fig. 5 shows the modification described in [3] for the wave variable method for the variable time-delayed teleoperation.

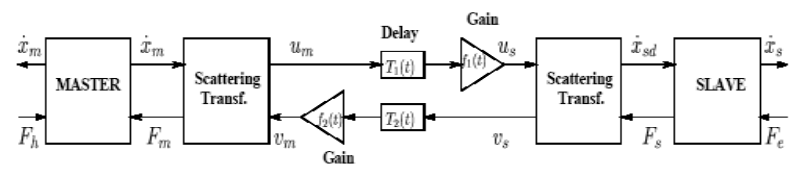

Fig. 5. Wave variable technique with adaptive gain component for teleoperation systems experiencing variable time delays [3]

Time varying delay modifies the transmission equations to:

$u_{s}(t)=u_{m}\left(t-T_{1}(t)\right) ; \quad v_{m}(t)=v_{s}\left(t-T_{2}(t)\right)$

where, $T_{1}(t)$ is the variable time delay in the path from the master to the slave and $T_{2}(t)$ is the variable time delay in the path from the slave to the master. In [3], it is assumed that the frequency of change in time delays remain limited:

$\frac{d T_{i}}{d \tau}<1 ; \quad i=1,2$

Substituting the modified transmission equations to the equation for the total energy stored in the communication line (5): 


$$
\begin{gathered}
E=\frac{1}{2}\left[\int_{t-T_{1}(t)}^{t} u_{m}^{T}(\tau) u_{m}(\tau) d \tau+\int_{t-T_{2}(t)}^{t} v_{s}^{T}(\tau) v_{s}(\tau) d \tau\right. \\
\quad-\int_{0}^{t-T_{1}(t)} \frac{T_{1}^{\prime}(\sigma)}{1-T_{1}^{\prime}(\sigma)} u_{m}^{T}(\sigma) u_{m}(\sigma) d \sigma \\
\left.-\int_{0}^{t-T_{2}(t)} \frac{T_{2}^{\prime}(\sigma)}{1-T_{2}^{\prime}(\sigma)} v_{s}^{T}(\sigma) v_{s}(\sigma) d \sigma\right]
\end{gathered}
$$

where $\sigma=\tau-T_{i}(\tau)=g_{i}(\tau)$ and $T_{i}^{\prime}(\sigma)=\left.\frac{d T_{i}}{d \tau}\right|_{\tau=g^{-1}(\sigma)}$.

The last two terms in (12) show that passivity can not be guaranteed for variable time-delayed teleoperation. In the modified wave variable method, shown in Fig. 5, a time varying gain $f_{i}$ is inserted after the time varying delay block. Therefore, the new transmission equation becomes:

$$
u_{s}(t)=f_{1}(t) u_{m}\left(t-T_{1}(t)\right) ; \quad v_{m}(t)=f_{2}(t) v_{s}\left(t-T_{2}(t)\right)
$$

The total energy stored can be re-written using the new transmission equations as

$$
\begin{gathered}
E=\frac{1}{2}\left[\int_{t-T_{1}(t)}^{t} u_{m}^{T}(\tau) u_{m}(\tau) d \tau+\int_{t-T_{2}(t)}^{t} v_{s}^{T}(\tau) v_{s}(\tau) d \tau\right. \\
-\int_{0}^{t-T_{1}(t)} \frac{1-T_{1}^{\prime}(\sigma)-f_{1}^{2}}{1-T_{1}^{\prime}(\sigma)} u_{m}^{T}(\sigma) u_{m}(\sigma) d \sigma \\
\left.-\int_{0}^{t-T_{2}(t)} \frac{1-T_{2}^{\prime}(\sigma)-f_{2}^{2}}{1-T_{2}^{\prime}(\sigma)} v_{s}^{T}(\sigma) v_{s}(\sigma) d \sigma\right]
\end{gathered}
$$

If $f_{i}$ is selected that $f_{i}^{2}=1-T_{i}^{\prime}$ for the total energy stored equation, the last two terms of the equation are eliminated and it can be said that the system is passive. In fact, the variable time-delayed system is considered to be passive if $f_{i}$ satisfies the following condition [3]:

$f_{i}^{2} \leq 1-\frac{d T_{i}}{d t} ; i=1,2$

This variable gain is called adaptive since it adapts itself with respect to the change in time delays.

\section{LIMITED-WORKSPACE TELEOPERATION EXPERIMENTS}

A commercial force-feedback joystick [10] is used as the master system and the Phantom Omni Device [11] is used as the slave system in this teleoperation experiment. The operator using the master system drives the slave in two axes. On the other side of the teleoperation, another operator holds the stylus of the Phantom Omni Device. Slave side operator is forced to follow the demands from the master. As the slave side operator resists following the demands, this resistance is recognized as the environmental forces and they are reflected to the master system. The rule to recognize the resistance is declared as feeding in a force more than $0.3 \mathrm{~N}$ to the Phantom Omni Device in any direction. The experimental setup is illustrated in Fig. 6.

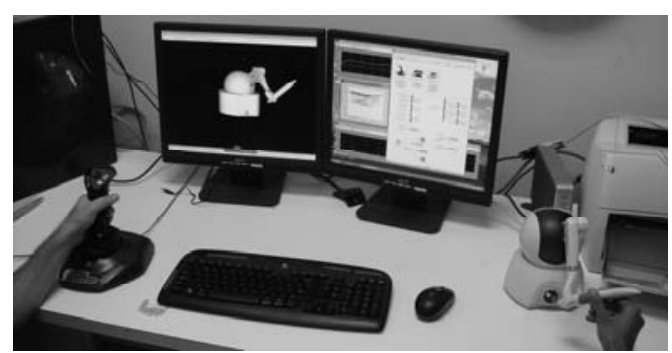

Fig. 6. Experimental setup for commercial joystick as the master and Phantom Omni Device as the slave

The experiments are performed at $1 \mathrm{kHz}$ sampling rate. The variable time delays and the adaptive gain variation due to the changes in time delays used in this experiment are shown in Fig. 7. The variable time delay profile is selected so that it is consistent with time delays over the Internet measured from the communication between Atlanta and Metz, France [2].

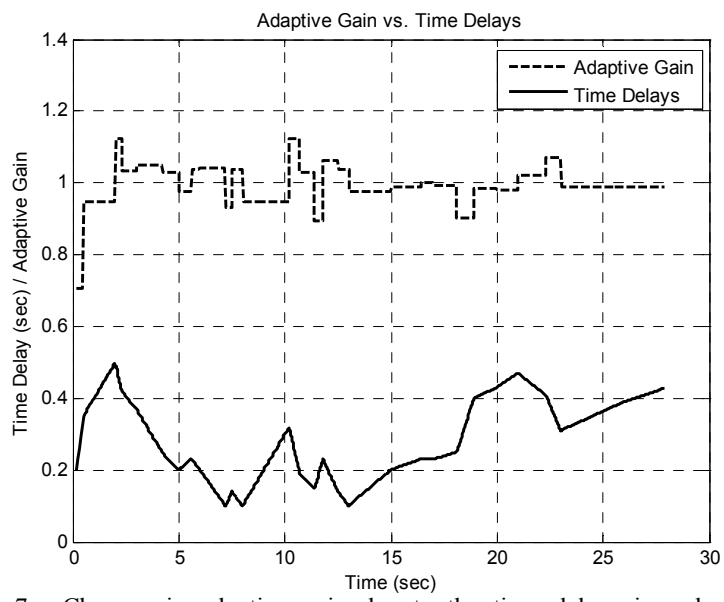

Fig. 7. Changes in adaptive gain due to the time delays in redundant teleoperation with the Logitech joystick and the Phantom Omni Device

The wave variable technique with position feedforward and adaptive gain is always the active controller throughout this experiment. The main reason for this is to protect the actual devices from excessive amounts of forces as the system is shown to be unstable with the other controllers in the previous experiment. The following figures present the position tracking performance of the master and the slave in this experiment.

It is observed from Fig. 8 that the system is stable but there are position errors in both axes. The reason for these errors is that the slave side is constantly under the control of the slave side operator. The operator resists to the motion demands as they are issued. The effects of this resistance are shown in 
Fig. 9 as the slave forces reflected back to the master. This figure provides a better idea on the position error observed in Fig. 8.

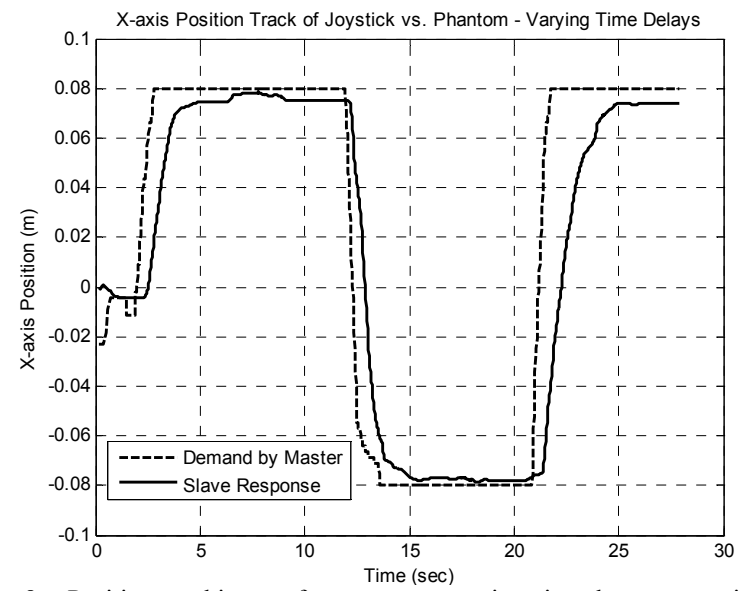

Fig. 8. Position tracking performance on $\mathrm{x}$-axis using the wave variable technique with position feedforward and adaptive gain components

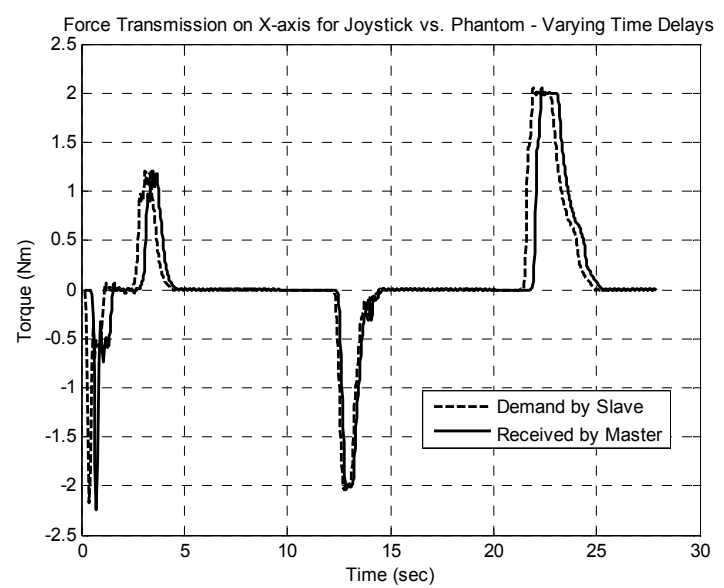

Fig. 9. Force tracking performance of the wave variable technique with adaptive gain and position feedforward components under variable time delays (x-axis)

It is clearly observed from Fig. 9 that the slave operator resists to the motion demands between the $22^{\text {nd }}$ and $25^{\text {th }}$ seconds. The slave forces increase as the slave system is forced not to follow the motion demands as observed in Fig. 8 between the $22^{\text {nd }}$ and $25^{\text {th }}$ seconds.

The results of this experiment show that the teleoperation system composed of actual robotic devices can be stabilized under time delays using the wave variable technique with position feedforward and adaptive gain components. The tracking performances are also shown to be satisfactory for both position and force demands.

\section{UNLIMITED-WORKSPACE TELEOPERATION EXPERIMENTS}

The commercial joystick used in the previous experiment and the actual holonomic mobile platform described in [12] are used to configure the teleoperation system for this experiment as shown in Fig. 10. The experiment is conducted at a sampling rate of $20 \mathrm{~Hz}$. The main reason for this is the communication speed limitations through the serial port of the computer. Although the sampling rate is lower than the previous experiments, teleoperation system is still able to operate since the mobile platform travels in relatively slow speeds $\left(\mathrm{V}_{\max }=80 \mathrm{~mm} / \mathrm{sec}\right)$. A source code working in the microprocessor of the mobile platform supplies the necessary outputs to drive the servomotors while obtaining the sensory information from the range sensors. An interface written in Matlab $^{\odot}$ M-file programming language is also created to send velocity demands to the microprocessor of the mobile platform and receive sensory information.
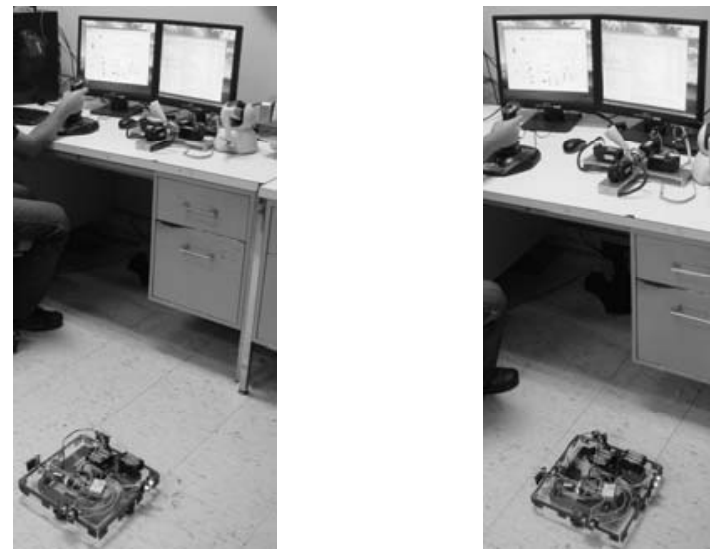

Fig. 10. Experimental setup for the master joystick and the holonomic mobile platform

The wave variable technique with adaptive gain component is used throughout the experiment. The following figures show the mobile platform approaching to obstacles. The first figure is drawn for the velocity tracking performance of the teleoperation system. The slave velocity shown in the figure is velocity demand received at the slave side from the communications line. The reasons for this are that the slave system does not have sensors to measure its velocity and the sonar sensor have limited range which makes them useless in measuring speeds when the system is not close to the obstacles.

Fig. 11 indicates that the system is stable and velocity tracking performance is satisfactory using the wave variable technique with the adaptive gain. Fig. 12 shows the force tracking performance of this teleoperation system as the slave approaches obstacles. The distance measured by using range sensors on the slave system is then converted to forces to be sent to the master. The forces are regulated as a result of this conversion and necessary amounts of torques are applied to the actuators of the joystick in order to make the human operator feel the presence of an obstacle.

It is clearly observed from Fig. 11 that the system is stable throughout the experiment and excessive amounts of forces are not formed as a result of any instability. Fig. 12 indicates that the force tracking performance is satisfactory along both axes using the wave variable technique with adaptive gain. 


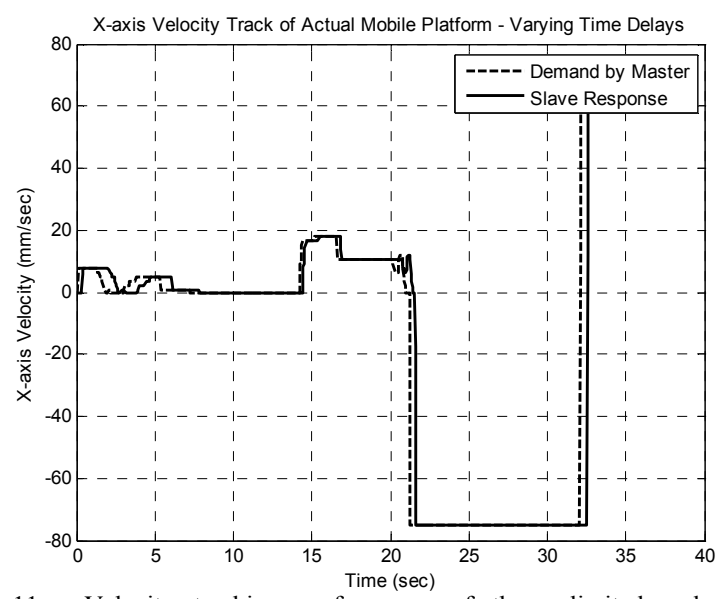

Fig. 11. Velocity tracking performance of the unlimited-workspace teleoperation with actual slave under variable time delays (x-axis)

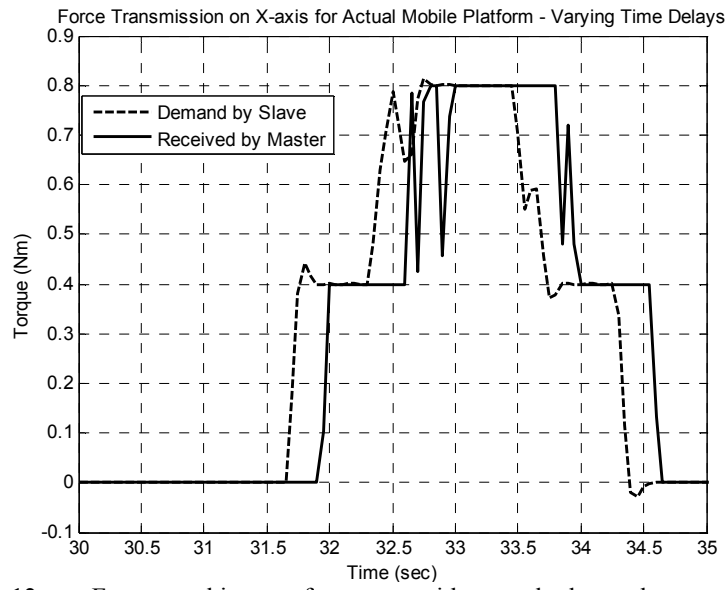

Fig. 12. Force tracking performance with actual slave along $\mathrm{x}$-axis (zoomed)

\section{CONCLUSIONS}

The experiments with limited-workspace teleoperation systems are conducted under variable time delays to observe the tracking performances and the stability. Previous work with constant time delays has shown that the customary wave variable technique guarantees stability under constant time delays. However, this controller fails under communication data loss failures in limited-workspace teleoperation. The feedforward position component has been shown to overcome this deficiency. Still the system is unstable when it is exposed to variable time delays. Hence, the adaptive gain component is added to the control scheme. Force tracking performance, on the other hand, was not affected in any of the experiments and therefore, a force feedforward component was found to be unnecessary in the controller architecture. The results were satisfactory as both components are used for unlimitedworkspace teleoperation systems examined under variable time delays.

The tracking priority is usually given to the velocity demand when unlimited-workspace slaves are used. Therefore, previous experiments with the customary wave variables provided satisfactory results even after a limited period of communication loss under constant time delays. In contrast, system becomes unstable under variable time delays. The addition of an adaptive gain component to the customary wave variable technique is shown to stabilize the system even under variable time delays. Therefore, the wave variable technique with adaptive gain component is recommended for unlimited-workspace teleoperation systems that experience variable time delays.

Overall, the teleoperation system designer first has to classify the system either as a limited- or unlimitedworkspace bilateral teleoperation. Then, the communications line conditions should be evaluated to see if the system will experience variable time delays or communication losses during the operation. As a result of this, wave variables technique with additional components can be used to configure a stable system without significant tracking errors. Thus, the end result becomes a more reliable system that can be applied to a wider range of critical missions.

The magnitude of time delays used in this work is consistent with the measured Internet delays. The range of delays should be increased in future work concentrating on large-scale time delays observed in teleoperation. This will provide better knowledge for space teleoperation or Mars/Lunar teleoperation missions that experience time delays in the range of hours.

\section{REFERENCES}

[1] G. Niemeyer, and J. Slotine, "Using Wave Variables for System Analysis \& Robot Control," Proceedings of the IEEE International Conference on Robotics and Automation, vol. 2, pp. 1619-1625, 1997.

[2] S. Munir, and W. Book, (2002). "Internet-Based Teleoperation Using Wave Variables with Prediction," IEEE/ASME Transactions on Mechatronics, vol. 7, is. 2, pp. 124-133. 2002.

[3] N. Chopra, M.W. Spong, S. Hirche, and M. Buss, "Bilateral Teleoperation over the Internet: the Time Varying Delay Problem," Proceedings of the American Control Conference, vol. 1, pp. 155-160, 2003.

[4] Jet Propulsion Laboratory, California Institute of Technology, California. Retrieved September 26, 2007 from http://wwwrobotics.jpl.nasa.gov.

[5] Foster-Miller, Inc., Massachusetts. Retrieved September 26, 2007 from http://www.foster-miller.com.

[6] R.J. Anderson, and W. Spong, "Bilateral Control of Teleoperation with Time Delay," IEEE Transaction on Automation and Control, vol. 34, is.5, pp.494-501, 1989.

[7] N. Chopra, M.W. Spong, R. Ortega, and N.E. Barbanov, "On Tracking Performance in Bilateral Teleoperation", IEEE Transaction on Robotics, vol. 22, is. 4, pp. 861-866, 2006.

[8] M.I.C. Dede, and S. Tosunoglu, "Modification of the Wave Variable Technique for Teleoperation Systems Experiencing Communication Loss," Proceedings of the $7^{\text {th }}$ IEEE International Symposium on Computational Intelligence in Robotics and Automation, pp. 380-385, 2007.

[9] M.K. Özgoren, "Topological Analysis of 6-Joint Serial Manipulators and Their Inverse Kinematics Solutions," Mechanism and Machine Theory, vol. 37, is. 5, pp. 511-547, 2002.

[10] Logitech. Retrieved September 26, 2007 from http://www.logitech.com.

[11] SensAble Technologies, Inc. Retrieved September 26, 2007 from http://www.sensable.com.

[12] M.I.C. Dede, and S. Tosunoglu, "Design of a Fault-Tolerant Holonomic Mobile Platform," Proceedings of the $19^{\text {th }}$ Florida Conference on Recent Advances in Robotics, 2006. 